\title{
Convective Turing Patterns
}

\author{
Desiderio A. Vasquez, ${ }^{1}$ Joseph W. Wilder, ${ }^{2}$ and Boyd F. Edwards ${ }^{1}$ \\ ${ }^{1}$ Department of Physics, West Virginia University, P.O. Box 6315, Morgantown, West Virginia 26506-6315 \\ ${ }^{2}$ Department of Mathematics, West Virginia University, P.O. Box 6310, Morgantown, West Virginia 26506-6310 \\ (Received 18 November 1992)
}

Turing patterns involve regions of different chemical compositions which lead to density gradients that, in liquids, are potentially unstable hydrodynamically. Nonlinear hydrodynamics coupled with a model of Turing pattern formation show that convection modifies and coexists with some Turing patterns and excludes others, and thereby plays a significant role in pattern selection.

PACS numbers: $47.20 . \mathrm{Bp}, 47.70 . \mathrm{Fw}, 82.20 . \mathrm{Mj}$

In 1952, Alan Turing proposed a chemical pattern formation mechanism based solely on a reaction-diffusion model [1]. It is believed that this mechanism plays a crucial role in biological morphogenesis, the development of pattern and form in the embryo. Recent chemical experiments have shown the existence of Turing patterns [2,3]. These patterns are formed when the homogeneous steady state loses stability and evolves into a state which consists of regions of different chemical composition. The resulting chemical gradients may lead to mass density gradients that render the patterns hydrodynamically unstable. For these reasons, the first experimental observations of Turing patterns took place inside a polyacrilamide gel designed to avoid fluid motion. Further experiments show that Turing patterns also exist in liquids confined in capillary tubes [3]. In the present work, we study the relation between Turing patterns and convection. This study is relevant to further experimentation in liquids and may be useful in understanding problems in areas where Turing pattern formation plays a role, such as biological morphogenesis. The importance of convection in chemical pattern formation has been determined in previous experimental and theoretical work involving chemical waves. In experiments on the iodate-arsenous acid reaction [4] and the Belousov-Zhabotinsky reaction [5], the speed of the chemical wave depends on the thickness of the chemical layer in petri dishes, thus indicating the presence of convection. It has been established that convection modifies the chemical front in vertical capillary tubes, from a flat convectionless front to a curved convective front $[4,6,7]$. These experiments indicate that convection plays an important role in chemical wave propagation and suggest that convection may also be important in Turing patterns.

We choose the standard Schnackenberg model as our model of Turing pattern formation [8,9] and couple it to the hydrodynamic equations of motion. Since the hydrodynamic parameters (density gradient, viscosity, etc.) have not been measured yet, we estimate them from the ones measured for the iodate-arsenous acid reaction. The uncertainty in having a complete set of parameters that corresponds to a particular system allows the use of the Schnackenberg model instead of a more realistic one.
The Schnackenberg model consists of four reaction steps:

$$
\begin{aligned}
& k_{1} \\
& A \rightarrow X \text {, } \\
& k_{2} \\
& X \rightarrow \text { products, } \\
& k_{3} \\
& 2 X+Y \stackrel{k_{3}}{\rightarrow} 3 X, \\
& k_{4} \\
& B \rightarrow Y \text {. }
\end{aligned}
$$

The pool chemicals $A$ and $B$ remain constant while $X$ and $Y$ obey the mass action law. We assume that the density $\rho$ varies linearly with the concentration $X$ :

$$
\rho=\rho_{0}\left[1-\alpha\left(X-X_{0}\right)\right] .
$$

Here, $\alpha$ is the coefficient of linear expansion and $\rho_{0}$ is the density when $X=X_{0}$, the reference concentration. In this work we assume that $\alpha$ is positive; consequently, the density is lower at higher concentrations of $X$. We neglect thermal expansion which may arise in exothermic reactions. The Schnackenberg model, coupled with hydrodynamics, provides the equations of motion for the system:

$$
\begin{aligned}
& \frac{\partial \mathbf{V}}{\partial t}+(\mathbf{V} \cdot \mathbf{\nabla}) \mathbf{V}=-\frac{1}{\rho_{0}} \nabla P+\frac{\rho-\rho_{0}}{\rho_{0}} \mathbf{g}+v \nabla^{2} \mathbf{V}, \\
& \mathbf{\nabla} \cdot \mathbf{V}=0, \\
& \frac{\partial X}{\partial t}+(\mathbf{V} \cdot \boldsymbol{\nabla}) X=D_{X} \nabla^{2} X+k_{1} A-k_{2} X+k_{3} X^{2} Y,
\end{aligned}
$$

and

$$
\frac{\partial Y}{\partial t}+(\mathbf{V} \cdot \boldsymbol{\nabla}) Y=D_{Y} \nabla^{2} Y+k_{4} B-k_{3} X^{2} Y .
$$

Here, $\mathbf{V}$ is the fluid velocity, $P$ is the standard reduced pressure which is related to the pressure $p$ by $P=p$ $+\rho_{0} g z, \mathbf{g}$ is the acceleration of gravity in the vertical $z$ direction, and $v$ is the kinematic viscosity. The density difference is included only where it modifies the large gravity term. We consider only two-dimensional motion confined to the $x-z$ plane. Since the velocity has zero divergence, we make use of the stream function $\psi$ and the vorticity $\omega$ to eliminate the reduced pressure. The com- 
ponents of the fluid velocity are related to the stream function and the vorticity by

$$
V_{x}=\frac{\partial \psi}{\partial z}, \quad V_{z}=-\frac{\partial \psi}{\partial x}
$$

and

$$
\omega=\nabla^{2} \psi .
$$

We introduce dimensionless variables

$$
\begin{aligned}
& t^{\prime}=\frac{v}{L^{2}} t, \quad \mathbf{x}^{\prime}=\frac{1}{L} \mathbf{x}, \quad d=\frac{D_{X}}{D_{Y}}, \quad \gamma=\frac{L^{2} k_{2}}{D_{X}}, \\
& a=\frac{k_{1}}{k_{2}}\left(\frac{k_{3}}{k_{2}}\right)^{1 / 2} A, \quad b=\frac{k_{4}}{k_{2}}\left(\frac{k_{3}}{k_{2}}\right)^{1 / 2} B, \\
& u=\left(\frac{k_{3}}{k_{2}}\right)^{1 / 2} X, \quad v=\left(\frac{k_{3}}{k_{2}}\right)^{1 / 2} Y, \\
& \mathbf{V}^{\prime}=\frac{L}{D_{X}} \mathrm{~V}, \quad P^{\prime}=\frac{L^{2}}{\rho_{0} D_{X^{v}}} P, \quad \psi^{\prime}=\psi / v, \quad \omega^{\prime}=\omega / v, \\
& \mathrm{Ra}=\frac{g \alpha L^{3}}{D_{X} v}\left(\frac{k_{2}}{k_{3}}\right)^{1 / 2}, \quad \mathrm{Sc}=\frac{v}{D_{X}} .
\end{aligned}
$$

The dimensionless parameter Sc is the Schmidt number and $\mathrm{Ra}$ is analogous to the Rayleigh number for thermal convection. The length $L$ is the typical length scale of the system to be defined later. With these substitutions, the governing equations become

$$
\begin{aligned}
& \frac{\partial \omega}{\partial t}=\frac{\partial(\psi, \omega)}{\partial(x, z)}+\frac{\mathrm{Ra}}{\operatorname{Sc}} \frac{\partial u}{\partial x}+\nabla^{2} \omega, \\
& \operatorname{Sc} \frac{\partial u}{\partial t}=\operatorname{Sc} \frac{\partial(\psi, u)}{\partial(x, z)}+\nabla^{2} u+\gamma\left(a-u+u^{2} v\right), \\
& \operatorname{Sc} \frac{\partial v}{\partial t}=\operatorname{Sc} \frac{\partial(\psi, v)}{\partial(x, z)}+d \nabla^{2} v+\gamma\left(b-u^{2} v\right), \\
& \omega=\nabla^{2} \psi
\end{aligned}
$$

where we have dropped the primes and define

$$
\frac{\partial\left(f_{1}, f_{2}\right)}{\partial(x, z)}=\frac{\partial f_{1}}{\partial x} \frac{\partial f_{2}}{\partial z}-\frac{\partial f_{2}}{\partial x} \frac{\partial f_{1}}{\partial z} \text {. }
$$

We here consider Turing patterns confined in rectangular boxes, demanding no chemical flow and no slip at the walls. The parameters $a, b$, and $d$ determine the stability of the homogeneous convectionless steady state. The set of parameters which leads to Turing pattern formation has been studied extensively elsewhere [8,9]. Since we are interested mainly in the effects of hydrodynamics on a well-developed pattern, we shall use a set of parameters that lead to a convectionless Turing pattern, namely, $a=0.14, b=1.1$, and $d=20$. In one dimension, the Turing pattern develops a characteristic wavelength. Choosing the length scale $L$ to approximate half the wavelength leads to $\gamma=40$. In the iodate-arsenous acid reaction the diffusion coefficient is $D=2 \times 10^{-5} \mathrm{~cm}^{2} / \mathrm{sec}$ and the kinematic viscosity is $v=9.2 \times 10^{-3} \mathrm{~cm}^{2} / \mathrm{sec}$; hence the
Schmidt number is 460 . In Turing patterns these parameters may be different; our calculations are carried out with $\mathrm{Sc}=200$ as a reasonable value. The above equations are solved numerically using a rectangular mesh. The mesh size is varied to address the computational needs of each particular case. The spatial derivatives are calculated using central differences and a five-point expansion is used to approximate the Laplacian. The time evolution is calculated using an alternate direction implicit scheme (ADI) [10], and the Poisson equation is solved using a relaxation technique [11].

A necessary condition for a steady convectionless Turing pattern can be obtained from the equations of motion with zero velocity and no time dependence [12],

$$
0=-\nabla P+\left(\rho-\rho_{0}\right) \mathbf{g} \text {. }
$$

Taking the curl, we obtain

$$
0=\mathbf{g} \times \nabla \rho
$$

so the density gradient must be in the same direction as the gravitational field. Any Turing pattern with a horizontal gradient must exhibit convection. All the experiments to date have observed only patterns with vertical and horizontal gradients. This fact is independent of the particular reaction-diffusion mechanisms that lead to the pattern, so the results obtained with the Schnackenberg model can be extended to other models.

We consider several cases of highly confined Turing patterns in rectangular boxes. The size of the boxes are

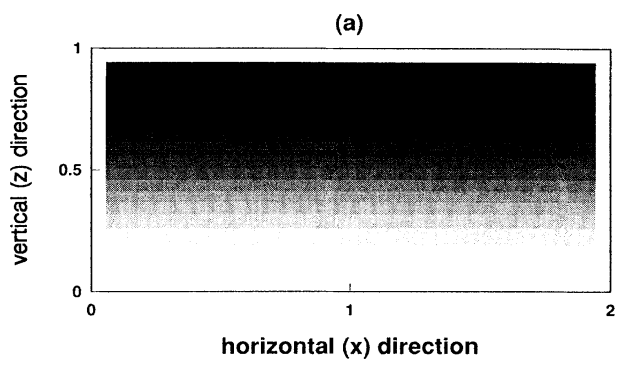

(b)

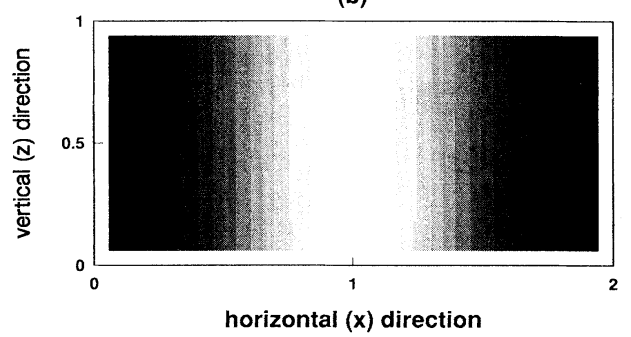

FIG. 1. Concentration of the variable $u$ for a Turing pattern confined to a $1 \times 2$ box; (a) without convection and with a chemical gradient in the vertical direction and (b) without convection and with a chemical gradient in the horizontal direction. The lighter regions represent higher concentrations of $u$ and lower mass densities. (Mesh size $36 \times 18$.) 
(a)

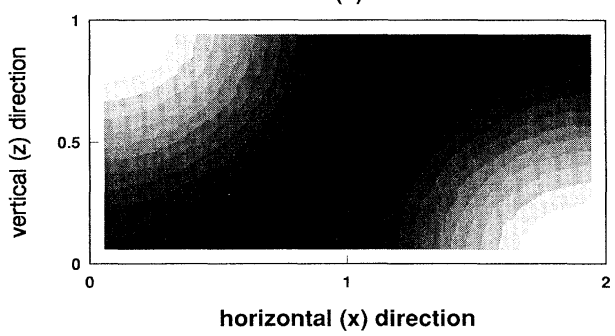

(b)

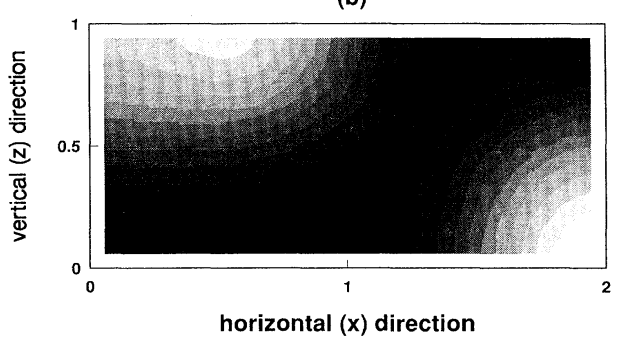

FIG. 2. Concentration of the variable $u$ for a Turing pattern confined to a $1 \times 2$ box; (a) no convection and chemical gradient in the vertical and the horizontal directions and (b) with steady convection. The lighter regions represent higher concentrations of $u$ and lower mass densities. ( $\mathrm{Ra}=60$; mesh size $36 \times 18$.)

of the order of a few wavelengths and the discretized equations on a rectangular mesh can be integrated using a reasonable amount of computer time. We do not treat extended Turing patterns because they will require a very large mesh and much longer computations. We first analyze Turing patterns in boxes of height 1 and length 2 in dimensionless units. In this case, we find three types of convectionless patterns (and their symmetric counterparts) with concentration profiles shown in Figs. 1(a), 1(b), and 2(a). To test their hydrodynamic stability, we study the time evolution of small random perturbations to the convectionless patterns. The convectionless state in Fig. 1 (a) is stable if the lighter fluid (higher values of $u$ ) is above the heavier fluid. The inverse configuration (light below heavy) is stable for $\mathrm{Ra} \leq 70$ and for $\mathrm{Ra} \geq 90$ the lighter fluid moves above the heavier and convection stops. The second pattern consists of three vertical regions [Fig. 1(b)]. As we previously discussed, because of the density gradient in the horizontal direction there is no steady convectionless state. Any value of the Rayleigh number will result in convection. This convection makes the pattern unstable and later becomes the first pattern with the lighter fluid on top. The third pattern has a density gradient in both the horizontal and the vertical directions [Fig. 2(a)]. In this case convection is always present as long as the Rayleigh number is different from zero. The system evolves with time into a steady convective state and modifies the Turing pattern as shown in Fig. 2(b). The convective pattern resembles the original pattern, but the upper and lower portions are no longer symmetric. The velocity field in this system consists of two

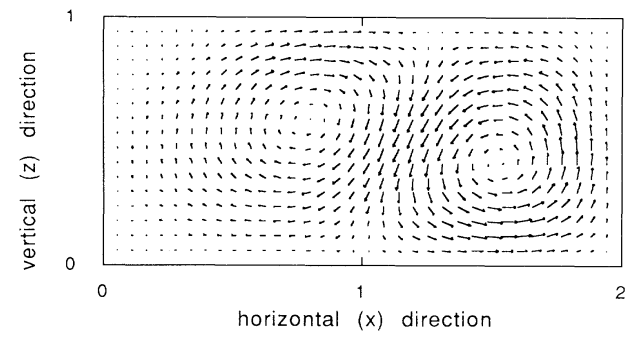

FIG. 3. Velocity field for a Turing pattern in a $2 \times 1$ box. The chemical concentrations correspond to Fig. 2(b).

convective rolls (Fig. 3) with fluid rising on the sides and falling in the middle. The rolls are not symmetric with the upper flow being stronger in the region where the lighter fluid is placed below the heavier fluid due to buoyancy. In this case, the distortion of the Turing pattern caused by convection is noticeable even for $\mathrm{Ra} \approx 20$. Convection is present for all Rayleigh numbers and consequently the distortion of the convectionless Turing pattern is always present.

In the iodate-arsenous acid reaction the fractional density difference is about $10^{-4}$; consequently we set its ana$\log \alpha\left(k_{2} / k_{3}\right)^{1 / 2}$ to this value. We estimate $\mathrm{Ra}=0.26$ using previously defined constants for the iodate-arsenous acid reaction and Turing pattern wavelength of $0.2 \mathrm{~mm}$ $\left(g=980 \mathrm{~cm} / \mathrm{sec}^{2}\right)$. For this estimate of the Rayleigh number, the distortion in the convective Turing pattern is small due to the small fluid velocity. If the Turing pattern wavelength (or the density gradient) increases by

(a)

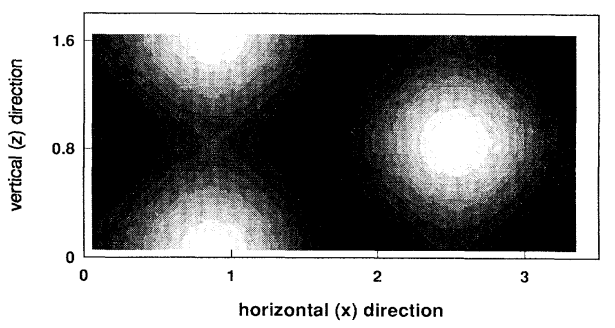

(b)

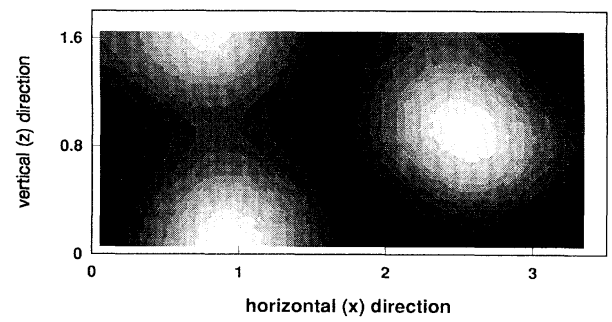

FIG. 4. Concentration of the variable $u$ for a Turing pattern confined to a $3.4 \times 1.7$ box; (a) without convection and (b) with convection. The lighter regions represent higher concentrations of $u$. $(\mathrm{Ra}=30$; mesh size $64 \times 32$. 


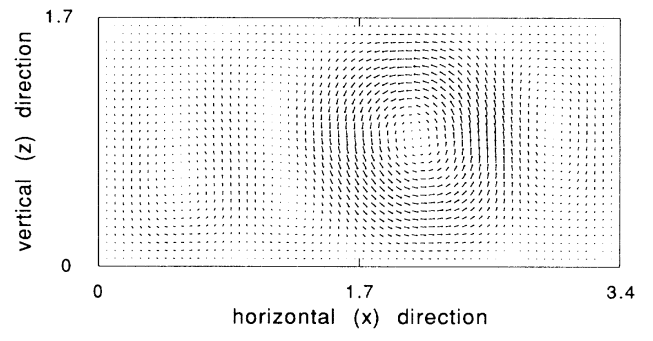

FIG. 5. Velocity field for a Turing pattern in a $3.4 \times 1.7$ box. The chemical concentrations correspond to Fig. 4(b).

changing the chemical composition of the mixture, then $\mathrm{Ra}$ increases and the fluid effects will become more important. If $\mathrm{Ra}>193$, the convective pattern evolves into a convectionless pattern with the lighter fluid above the heavier fluid, as in the case of the second pattern.

We have also considered a box of dimensionless size $3.4 \times 1.7$ which accommodates a more extended pattern. Here we restrict our attention to one of a number of possibilities for Turing patterns in this geometry. In the convectionless case [Fig. 4(a)], there is a symmetry about a horizontal line in the middle of the box. This symmetry makes the lower and upper boundaries indistinguishable; we cannot recognize which way is up. For $\mathrm{Ra}=30$, there is a significant distortion due to the rising lighter fluid [Fig. 4(b)]. Convection is always present due to the gradient in the horizontal direction, with stronger convection for larger Rayleigh numbers. The fluid velocity field is shown in Fig. 5; the velocities are higher at the right side of the box causing the distortion of the circular region. Higher values of $u$ are found at the upper boundary since higher values of $u$ correspond to lighter fluid.

We have shown here the effects of hydrodynamics in Turing patterns based on the ideal Schnackenberg model. We found that not only can convection coexist with Turing patterns, but that it can also alter them significantly and can exclude some patterns predicted by the reactiondiffusion theory. We have shown that certain patterns will evolve into other patterns. This result indicates that convection plays an important role in pattern selection. This is important for Turing pattern formation with growing domains, which have an impact on biological problems. It has been shown that for reaction-diffusion models, the shape of the fully grown domain depends on the shape of the initial domain [9]. When convection is allowed, the pattern in the grown domain will evolve into another type of Turing pattern that can sustain convection. In more extended patterns, the fluid effects might become more important because the density gradient in the horizontal direction precludes a convectionless state. The effect is still small for estimates based on the iodate- arsenous acid reaction. However, the fluid velocity alters the patterns at relatively low Rayleigh numbers. New experiments in this regime might be designed to test these effects. The asymmetry caused by convection should be observable and may be tested by physically inverting the device. Since the Rayleigh number is proportional to the wavelength to the third power, fluid effects will become important for larger wavelengths. For this reason it may be difficult to observe patterns of larger wavelengths in liquids since the fluid effects are stronger. Buoyancy is an important mechanism in pattern selection as certain patterns cannot exist if the density gradient is too large.

Further studies in convective Turing patterns may yield oscillatory convection if the fluid density varies with both chemical concentrations and temperature. It may also play an important role when chemical turbulence takes place in liquid solutions. It will also be of interest in studying the effects of gravitational fluid motion on a recent model of chemical pattern formation [13] based on differential fluid flow.

Discussions with Kenneth Showalter are gratefully acknowledged. This work is supported in part by the $\mathrm{Na}$ tional Science Foundation Grant No. RII-8922106 and the National Research Center for Coal and Energy.

[1] A. M. Turing, Philos. Trans. R. Soc. London B 327, 37 (1952).

[2] V. Cassets, E. Dulos, J. Boissonade, and P. De Kepper, Phys. Rev. Lett. 64, 2953 (1990).

[3] K. Agladze, E. Dulos, and P. De Kepper, J. Phys. Chem. 96, 2400 (1992).

[4] T. McManus, Ph.D. thesis, West Virginia University, 1989.

[5] J. Rodriguez and C. Vidal, J. Phys. Chem. 93, 2737 (1989).

[6] J. A. Pojman, I. R. Epstein, T. J. McManus, and K. Showalter, J. Phys. Chem. 95, 1299 (1991).

[7] D. A. Vasquez, B. F. Edwards, and J. W. Wilder, Phys. Rev. A 43, 6694 (1991); B. F. Edwards, J. W. Wilder, and K. Showalter, Phys. Rev. A 43, 749 (1991).

[8] V. Dufiet and J. Boissonade, J. Chem. Phys. 96, 664 (1992).

[9] J. D. Murray, Mathematical Biology (Springer-Verlag, Berlin, 1989).

[10] J. K. Platten and J. C. Legros, Convection in Liquids (Springer-Verlag, Berlin, 1984).

[11] W. Press, B. Flannery, S. Teukolsky, and W. Vetterling, Numerical Recipes (Cambridge University Press, Cambridge, 1986).

[12] A. J. Pearlstein, J. Phys. Chem. 89, 1054 (1985).

[13] A. B. Rovinsky and M. Menzinger, Phys. Rev. Lett. 69, 1193 (1992). 
(a)

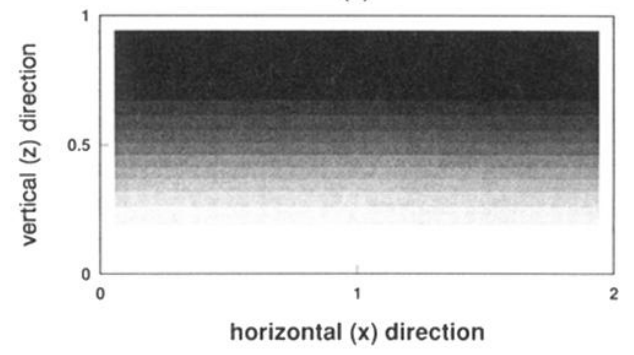

(b)

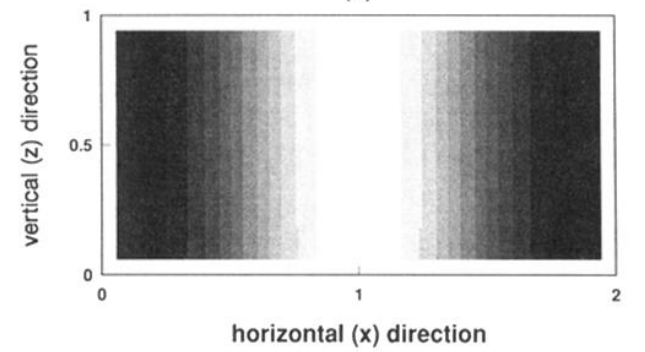

FIG. 1. Concentration of the variable $u$ for a Turing pattern confined to a $1 \times 2$ box; (a) without convection and with a chemical gradient in the vertical direction and (b) without convection and with a chemical gradient in the horizontal direction. The lighter regions represent higher concentrations of $u$ and lower mass densities. (Mesh size $36 \times 18$.) 
(a)

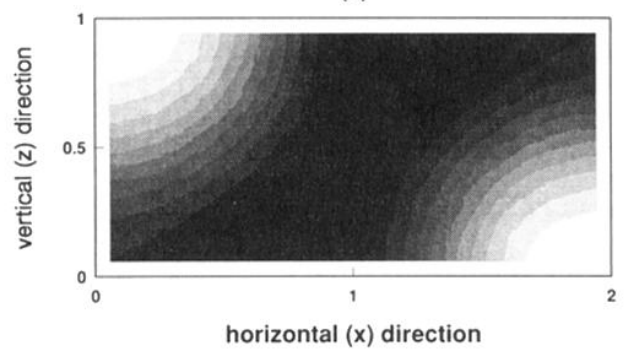

(b)

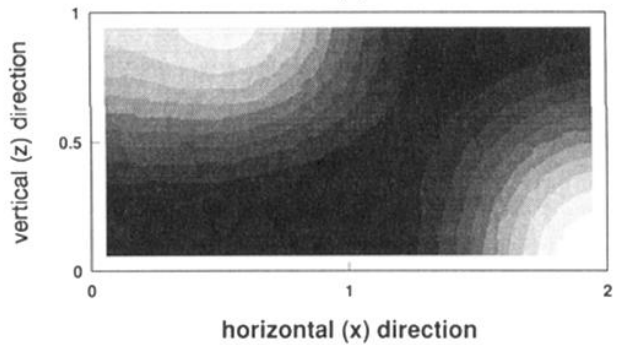

FIG. 2. Concentration of the variable $u$ for a Turing pattern confined to a $1 \times 2$ box; (a) no convection and chemical gradient in the vertical and the horizontal directions and (b) with steady convection. The lighter regions represent higher concentrations of $u$ and lower mass densities. $(\mathrm{Ra}=60$; mesh size $36 \times 18$.) 
(a)

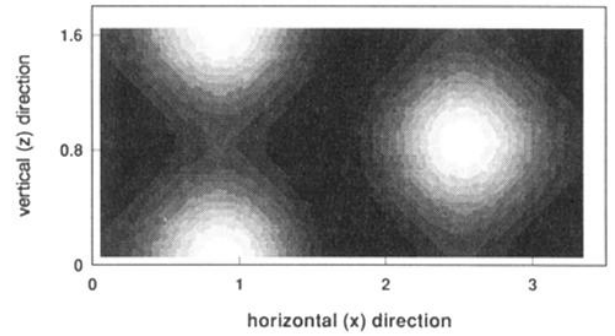

(b)

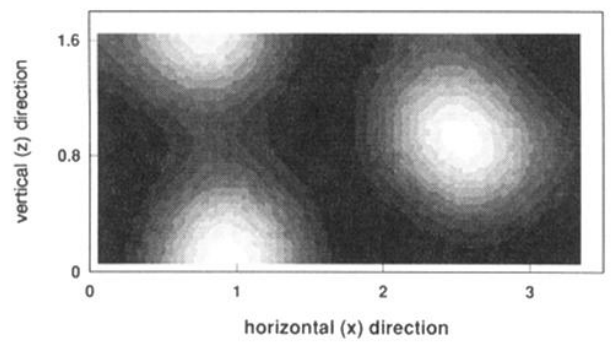

FIG. 4. Concentration of the variable $u$ for a Turing pattern confined to a $3.4 \times 1.7$ box; (a) without convection and (b) with convection. The lighter regions represent higher concentrations of $u$. $\quad(\mathrm{Ra}=30$; mesh size $64 \times 32$. $)$ 